\title{
L'ESPOIR ET SIERRA DE TERUEL, LA CHUTE ET LES REGARDS'
}

\author{
VINCENZO BORLIZZI \\ SCRIPT/RASM - Université d'Evry
}

\begin{abstract}
Résumé
Ce texte vise à approfondir la question de la chute des républicains espagnols et de sa représentation dans l'œuvre complexe entre roman et film d'André Malraux. Cette question est traitée sous trois axes: d'abord la forme de la déposition au début du film, après la chute d'un avion et la mort d'un des aviateurs; ensuite les rapports entre les chutes des corps dans le combat et la présence silencieuse d'une série de natures mortes englobant les gestes éphémères des êtres humains dans leur atemporalité ; finalement la forme de la descente collective des blessés à la fin du film et du roman et l'expression visuelle de la réminiscence de la tradition picturale et cinématographique. Ces trois chemins de réflexions amènent vers une pensée visuelle exprimant la chute d'un peuple comme un mouvement de descente vers le haut, un mouvement descendant à la deuxième puissance, comme Simone Weil avait proposé, où la pesanteur morale du combat de la liberté trouve une forme pour exprimer l'élan vers le haut.
\end{abstract}

Mots clés: Malraux, Adaptation, Chute, Déposition, Cadrage, Nature morte.

\begin{abstract}
This article aims to go into the issue of Spanish Republicans' fall in depth and to deepen the subject of representation of this fall in André Malraux novel and film. This issue is dealt with three axes : first of all, the form of Deposition at the film's beginning, after a plane's fall and the death of an aviator; later, the connections between the fall of human bodies in the struggle and the silent presence of some still lives which embrace short-lived acts of human beings into their timelessness; finally the form of casualties' collective descent of at the end of the film and of the novel in relation to the visual expression of reminiscence in pictorial and cinematic tradition. These three ways of reflection bring about a visual thought which formulates the fall of a nation as a movement of descent to the top, as a descending movement multiplied to the square exponentiation, like Simone Weil suggests, where moral gravity of the struggle for liberty finds a form to express a run up to the top.
\end{abstract}

Key words: Malraux, Adaptation, Fall, Act of creating cinema, framing, Still life.

1 SCRIPT/RASM Université d'Evry. Correo: vinceandzoo@gmail.com. Recibido: 26-01-2012. Aceptado: 20-04-2012. 
«-Que peux-tu faire pour un mort ?

- Lui être reconnaissant.»

Dialogue à la fin de Sierra de Teruel.

Barcelone, 2006. Un homme erre dans les rues, il cherche avec une caméra. Il cherche à trouver des images par d'autres images, il cherche les lieux des images qui sont pour lui le début de son rêve de cinéaste. Un homme prend la parole, c'est la voix de Victor Erice qui plane doucement sur la ville, par les «calles» et dans le vent, jusqu'à travers la Sierra, pour s'arrêter dans le village de la fin du film qu'il recherche². Oui, il recherche, car il tourne les mêmes plans du film qu'il cherche, il les retourne dans les mêmes endroits du film qui l'a poussé au tournage, comme quelqu'un qui cherche la mémoire d'une ou de plusieurs images par la répétition des gestes qui les ont précédées dans les mêmes lieux.

\section{Quel film recherche-t-il?}

Sierra de Teruel, œuvre inachevée, qui se cherche elle-même, tournée au milieu de mille difficultés et dans les aléas de la guerre civile espagnole, entre les mois d'août 1938 à janvier 1939, un film non accompli, réalisé par un cinéaste qui ne tournera plus jamais, André Malraux, aidé par un écrivain, Max Aub, qui ensuite dialoguera souvent avec un autre réalisateur, Luis Buñuel, le réalisateur qui, à un moment de sa vie, avait décidé d'arrêter la réalisation pour y revenir des décennies plus tard.

Ce film est affaire d'abandons, de longues pauses, d'années et de décennies qui s'écoulent entre un départ et un retour. Le film ne sera montré qu'en France, en séance privée pour le gouvernement espagnol en exil, avant le déclenchement de la guerre mondiale. Les hitlériens détruiront systématiquement toute copie du film. Une copie, une seule, car ce film est aussi question d'unicité, sera sauvée, et en 1945 le film sera montré en public, précédé par une brève allocution de Maurice Schumann. L'Espagne, la terre de la Sierra, ne connaîtra les visages de cet espoir perdu qu'en 1977.

Mais le film même est l'incarnation d'un livre, ou plutôt une auto-transposition d'un texte écrit par Malraux lui-même entre 1936 et les premiers mois de 1937 en Espagne ${ }^{3}$.

2 Il s'agit de Sierra de Teruel, 1939 (Espagne, 2006, 19'), deuxième chapitre de la série documentaire «Mémoire et rêve», série en cours de réalisation, produite, écrite, réalisée et commentée par Victor Erice. Jusqu'à présent, quatre chapitres ont été tournés: au-delà de ce deuxième chapitre, le premier est consacré à Rome Ville Ouverte de Rossellini, le troisième au Mépris de Godard et le quatrième à Mizoguchi Kenji. La partie dédiée au film de Malraux a fait l'objet d'une projection publique lors de la soirée d'ouverture de la programmation «Trafic - 20 ans, 20 films» au Centre Pompidou, à Paris le 11 janvier 2012, en honneur des vingt ans de la revue Trafic.

3 Le livre a été publié en décembre 1937, mais nous utiliserons l'édition Folio Gallimard de 1963. Nous joignons un tableau synoptique : il s'agit d'une segmentation du film, visant à éclaircir la structure du long-métrage par rapport au roman. Nous n'avons pas suivi le schéma «Film, Roman, Découpage», Annexe 2 du numéro de l'Avant-scène Cinéma (n³85, Octobre 1989:12-13), parce que il est inexact sous plusieurs aspects, notamment dans le travail des références du film au roman : par exemple, la première scène du film de Marcelino est l'union de plusieurs chapitres du livre, mais ce schéma n'indique qu'un 
Ce livre et ce film sont la sublimation littéraire et visuelle d'une chute et la démonstration que la grâce peut être atteinte par la chute corporelle dans le sacrifice d'une guerre perdue. Cet ouvrage, littéraire et filmique à la fois, est aussi la démonstration visuelle de l'idée soutenue par Simone Weil, l'autre combattante française dans la même guerre d'Espagne, aux côtés des républicains, la combattante de la France Libre plus tard (en rejoignant, là aussi, le résistant André Malraux), la jeune philosophe qui écrira de la grâce consistant en une chute à la deuxième puissance.

Ce texte ne vise qu'à démontrer cela: l'œuvre littéraire de Malraux se répand dans l'oeuvre filmique pour donner forme sonore et visuelle à cette chute multiple se transformant en grâce. Mais quel est l'enjeu, quel est le sens de cette forme visuelle de la chute?

L'hypothèse est que, face à la défaite et au martyre d'une grande partie du peuple espagnol, Malraux a voulu sublimer ce sacrifice par la conscience que toute chute précède un envol, et que l'attente du peuple se relevant a déterminé le caractère inachevé de l'œuvre. Il nous plaît d'imaginer le vieux Malraux allant terminer son film après la mort de Franco, entre la fin de l'année 1975 et le début de 1976, juste quelques mois avant de s'éteindre, un an après le dictateur qu'il a combattu.

Simone Weil introduit la problématique de la chute des corps entre la pesanteur et la grâce ainsi :

Descendre d'un mouvement où la pesanteur n'a aucune part...La pesanteur fait descendre, l'aile fait monter : quelle aile à la deuxième puissance peut faire descendre sans pesanteur ?

La création est faite du mouvement descendant de la pesanteur, du mouvement ascendant de la grâce et du mouvement descendant de la grâce à la deuxième puissance.

La grâce, c'est la loi du mouvement descendant.

S'abaisser, c'est monter à l'égard de la pesanteur morale. La pesanteur morale nous fait tomber vers le haut. (Weil, $1947: 10$ )

Est-il possible de créer l'image d'un peuple qui se relève tout en tombant? Est-il possible de transformer la chute dans un mouvement ascensionnel, ne fût-ce symbolique? Voici les problèmes que notre hypothèse essayera d'approfondir, en suivant trois parcours dans les images entre le livre et le film: la transformation de la chute d'un avion au début du film et de la mort d'un des aviateurs en une expression de sublimation figurative du corps allongé du mort; la présence visuelle d'éléments faisant référence aux natures mortes par rapport à l'action du combat, de la chute et de la mort; la transformation de la chute de l'avion et de la descente des blessés à la fin du roman et du film dans l'expression accomplie de cette idée de chute vers le haut.

seul chapitre au début de l'œuvre littéraire. En outre, nous avons décidé de nous tenir au film tel qu'il a été réalisé et monté, sans prendre en compte les scènes prévues et jamais tournées. 


\section{MARCELINO: UNE DÉPOSITION ET UNE NATIVITÉ.}

Le film s'ouvre avec une chute, celle d'un avion atteint par l'ennemi, et par la mort de Marcelino Rivelli, pilote italien dans l'escadrille internationale des républicains espagnols.

Lors de la vision de cette deuxième scène du film, ce qui frappe est la déposition picturale du corps de Marcelino dans la salle du terrain d'aviation. En tournant cette scène, l'écrivain ne pouvait pas ignorer les œuvres d'art qu'il citera dans la troisième partie de son ouvrage de 1951, Les voix du silence (Malraux, 2004:660 suiv.), dédiée à la création artistique, et traitant de l'Enterrement du comte d'Orgaz du Greco, dont il mettra en évidence le fragment du corps du comte déposé en horizontal, et qu'il associera à $\mathrm{d}^{\prime}$ autres œuvres du même peintre, surtout les ascensions verticales du Christ ${ }^{4}$.

Nous ne pouvons pas savoir si Malraux était déjà conscient en 1938 de l'association visuelle, et pourtant paradoxale, qu'il proposera dans son texte de 1951 entre la déposition en horizontal après la mort et l'ascension après la résurrection, comme si l'élévation pouvait aussi passer par le bas, voire comme si l'acte de Déposition était en quelque sorte un passage figuratif nécessaire pour arriver à l'ascension. Mais la question de cette conscience se pose notamment si nous revenons au livre de 1937, avant de passer au film et aux tableaux, et si nous voyons ensuite comment Malraux adapte et synthétise dans cette scène du film tout ce qui concerne Marcelino dans le roman.

Les références à Marcelino dans le livre sont éparpillées dans plusieurs chapitres : dans le troisième Chapitre de la sous-section II de la première Section de la Première Partie de l'œuvre, dont l'intitulé est «L'illusion lyrique» (Malraux, 1937: 7980), Magnin, le chef de l'escadrille de l'aviation (alter ego de Malraux, qui deviendra l'espagnol Peña dans le film) apprend l'histoire de la vie de Revelli, son envol au-dessus de Milan pour lancer des tracts contre le régime fasciste et ses années passées dans les prisons fascistes ; une autre brève référence est donnée pendant la description du chef espagnol de l'aviation interne, Sembrano, dont Malraux écrit qu' «il était naturellement courageux, comme Marcelino et comme tant de timides» (Malraux, 1937: 105).

En outre, une anticipation de la mort de Marcelino peut être vue dans le deuxième Chapitre, sous-section I, de la Section II (dénommée «Exercice de l'Apocalypse») de cette Première Partie du livre, quand l'historien de l'art italien Scali, rattaché à l'escadrille internationale d'aviation, tombe sur les papiers d'un pilote d'avion fasciste mort et d'un autre Italien, capturé vivant, dans le même avion fasciste, dénomme l'observateur, dans les locaux de la Sûreté de Madrid :

C'était la première fois que Scali rencontrait un Italien ennemi avec une illusion d'intimité, et celui qu'il rencontrait était un mort. (Malraux, 1937: 137)

4 Cette œuvre de Malraux renvoie plusieurs fois à la composition de la Déposition, notamment dans la première partie, le célèbre " Musée de l'imaginaire », quand il est question de la Déposition des Heures de Rohan de la première moitié du $15^{\text {ème }}$ siècle (Malraux, 2004: 225-229), ou de la figure étendue dans La mort de Procris de Piero di Cosimo (Malraux, 2004: 249), ou surtout à propos de l'esquisse pour la Pietà de Delacroix (Malraux, 2004: 310 suiv.). 
Parmi ces papiers, il y a une «petite photo [...], c'était un détail d'une fresque de Piero de la Francesca» (Malraux, 1937: 140), dont Scali est un spécialiste car «il avait publié l'analyse la plus importante des fresques de Piero.» (Malraux, 1937: 141). Scali découvre que la photo appartient au prisonnier italien vivant et il pense: «Sans le fascisme, cet homme êut peut-être été son élève» (Malraux, 1937: 141).

Mais ce qui est étonnant est la phrase qui suit:

«Scali avait pensé un instant que la photo avait appartenu au mort, dont il s'était senti confusément solidaire». (Malraux, 1937: 141)

Cette anticipation se passe donc trois chapitres avant la mort de Marcelino, qui arrive dans le cinquième chapitre de la même sous-section. Mais il y a également deux autres éléments : parmi les papiers du mort, Scali trouve un plan d'attaque aérienne sur Tolède pour le surlendemain et avertit tout de suite le commandant García. La conséquence narrative est le chapitre suivant (le troisième) où on retrouve Marcelino dans les fonctions de bombardier sur un avion républicain, dirigé vers Tolède pour se battre contre l'aviation fasciste et destiné à la mort à la fin du combat. En quelque sorte, le papier du mort engendre la mort de Marcelino.

Mais le deuxième élément qui lie Scali au prisonnier italien encore vivant n'appartient pas à l'aviateur fasciste mort, il s'agit d'une photo présentée par Scali au prisonnier comme en échange de la fresque de Piero (Malraux, 1937: 142): une photo du visage d'un républicain, le capitaine Vallado, qui avait participé à la prise de la caserne de la Montagne à Madrid (personnage cité dans le troisième Chapitre de la soussection I de la Section «L'illusion lyrique»), qui ensuite avait fait partie de l'aviation républicaine, et avait été abattu par les fascistes et torturé jusqu'à l'énucléation des yeux. Scali présente cette photo à son compatriote ennemi pour montrer ce que les fascistes faisaient aux ennemis. Mais justement cette image du visage sans regard va remplacer ce détail de la fresque de Piero, détail aveugle lui aussi pour le lecteur.

La question du regard et de cette anticipation de la chute et de la mort de Marcelino va davantage se compliquer avec le décès de ce dernier, trois chapitres plus loin, dans le cinquième Chapitre, sous-section I, Section II de la Première Partie :

Trois blessés, trois morts, dont Marcelino: six, il manquait un mitrailleur. C'était Jaime, qui descendit bien après les autres. Les mains en avant, qui tremblaient, et un camarade pour le guider: une balle explosive à al hauteur des yeux. Aveugle.

Par les épaules et par les pieds, les aviateurs portèrent les morts au bar. Le fourgon viendrait plus tard. Comme Marcelino avait été tué d'une balle dans la nuque. Il était peu ensanglanté. Malgré la tragique fixité des yeux que personne n'avait fermés, malgré la lumière sinistre, le masque était très beau.

L'une des serveuses du bar le regardait.

Il faut au moins une heure pour qu'on commence à voir l'âme, dit-elle.

Magnin avait vu assez mourir pour connaître l'apaisement qu'apporte la mort sur beaucoup de faces. Plis et petites rides étaient partis avec l'inquiétude de la pensée ; et devant ce visage lavé de la vie, mais où les yeux ouverts et le serre-tête de cuir maintenaient la volonté, Magnin pensait à la phrase qu'il venait d'entendre, qu'il avait entendue sous tant de formes en Espagne ; 
c'est seulement une heure après la mort, que, du masque des hommes, commence à sourdre leur vrai visage. (Malraux, 1937: 162)

Après un examen du travail de synthèse opéré au niveau du scénario, on observe que les premières deux scènes du film (Premier Segment, parties 1.A et 1,B) condensent toute ces références dans la chute de l'avion et dans la déposition du corps de Marcelino. Mais ce qui nous intéresse ici n'est pas l'expression de la chute aérienne, mais son résultat visuel le plus consistant, ce qui se passe dans la deuxième scène (1.B).

Si on regarde le douzième plan du film, avec le corps de Marcelino suspendu à quelques centimètres du sol, allongé en horizontal, face à ces figures verticales qui lui rendent hommage, la référence au tableau du Greco est évidente et nous pouvons lier cette disposition du corps tombé horizontalement à la fuite des anges au sommet du tableau (à préfigurer une ascension) et aux mots de la serveuse, répétés dans le film aussi, qui soulignent une ascension du visage, de la figure de l'aviateur par sa mort, retrouvant par là la dialectique entre l'horizontalité et la verticalité des Voix du Silence.

Cette hypothèse est étayée par nombre d'autres tableaux de la tradition occidentale qui expriment la déposition du Christ, notamment les deux œuvres parmi les plus connues de Giotto, celles de la Déposition de la Croix et de la Mort de François. Mais il n'est pas hasardeux d'avancer une autre réflexion, concernant la référence à Piero della Francesca dans le livre : Malraux ne nomme jamais le détail de la fresque dont Scali trouve un fragment dans les poches du prisonnier fasciste. Est-il possible que cette posture du corps déposé dans le film lui a été suggérée par la partie centrale de la fresque de la Mort d'Adam, fresque ruinée, fragmentée et qui préfigure un montage cinématographique de deux phases de la vieillesse d'Adam ? Cette fresque présente sur la droite Adam encore vivant, tandis que, au centre, le même Adam est allongé par terre, mort, avec la figure de Seth, dieu égyptien du chaos, qui pose le grain de l'arbre du péché dans la bouche du vieillard.

Considérons aussi deux tableaux de nativité dépeints par Piero, où la figure de l'enfant est positionné en horizontal par rapport aux autres figures verticales: estce que Malraux avait à l'esprit cette coïncidence horizontal/vertical entre la mort du premier homme et la naissance du rédempteur chez Piero? Cette concordance se fera plus complexe dès qu'on la met en relation avec la verticalité de la métaphore de la Croix du Christ. Est-ce que l'écrivain/cinéaste a voulu en quelque sorte suggérer une synthèse entre la fuite vers le haut des tableaux du Greco et cette dialectique vie/mort qui se passe dans les œuvres de Piero dans la posture horizontale du corps mort et renaissant?

L'interprétation est peut-être excessive, mais il n'en reste pas moins que ce nombre d'indices ici présents permettent de renforcer notre hypothèse quant à la transformation visuelle de la chute des combattants pour la liberté en une chute vers le haut, vers la renaissance d'un mouvement descendant, soutenu par la pesanteur éthique du geste et du mouvement vers la grâce, qui coïncide ici avec la liberté. Ce mouvement s'appuie enfin sur l'acte de ne pas voir du mort, les yeux fermés correspondant au sacrifice du républicain privé de ses yeux, torturé par les fascistes et 
présenté en photo dans le roman, comme un premier essai de photogramme du film envahissant le littéraire. La fin du regard correspondrait ainsi à une séparation de la pesanteur corporelle pour acquérir le «vrai visage »surgissant une heure après la mort et s'offrant dans un instant aux yeux ouverts des vivants restés là-bas, débout, tandis que l'ascension se passe à même le sol, par le bas ${ }^{5}$.

\section{LES NATURES MORTES: L'INDIFFÉRENCE DE L'ÉTERNITÉ ET DE LA NATURE.}

Cette image de la photographie du visage sans yeux ouvre aussi un deuxième chemin d'exploration de la question posée au début. Cette fois-ci il ne s'agit pas des hommes, mais de tout ce qui est autour d'eux, avec une temporalité complètement différente dans le livre et dans le film. Les mots de l'écrivain lient tout de suite le dialogue de Scali et de son compatriote sur la photographie du torturé avec cette dimension extrahumaine, dans un tas d'objets hétéroclites cumulés dans les salles de la Sûreté:

Il s'arrêta. De l'amoncellement d'argenterie, sortirent, comme des souris, un, deux, trois, quatre coups, aussi argentins et légers que s'il fussent venus non de quelque pendule enfouie dans ce bric-à-brac tragique, mais de ces trésors d'Aladin eux-mêmes. Ces pendules - remontées pour combien de temps ? - qui au milieu de cet entretien, si loin de ceux qui les avaient possédées, sonnaient une heure quelconque, donnaient à Scali une telle impression d'indifférence et d'éternité ; tout ce qu'il disait, tout ce qu'il pouvait dire lui sembla si vain qu'il n'eut plus envie que de se taire. Cet homme et lui avaient choisi. (Malraux, 1937: 143)

Dans le roman de Malraux, il y a une série de descriptions se rapprochant à celle-ci: ces expressions mettent en évidence une vie sécrète des objets et des éléments extrahumains, à côté des événements de la guerre. Il ne s'agit pas d'un hasard si plusieurs chapitres du livre (les chapitres 4,5 et 7 de la deuxième sous-section de $1^{\prime}$ «Exercice de l'Apocalypse») se passent dans le musée de Tolède, où les républicains attendent entre la bataille et l'armistice, avant la déroute et avant la prise de la ville de la part des franquistes: pour Malraux, l'occasion est propice pour présenter plusieurs descriptions d'objets qui ne sont pas de véritables tableaux de natures mortes, mais en réalité les descriptions mêmes deviennent l'expression d'un nombre de natures mortes à côté des combattants.

Voici deux exemples dans le texte:

Tous mangeaient. Dans les vitrines modernes du musée, verre, acier et aluminium, tout était en ordre, sauf de petits objets pulvérisés sur place par les balles un trou net entouré de rayons dans le verre devant eux.(Malraux, 1937: 199)

Les assiettes et les carafes à col d'alambic réverbéraient comme des vers luisants les milles points de lumière briques trouées, à travers l'énorme nature morte. Le long des branches brillaient les fruits, et les courtes lignes bleuâtres des canons des revolvers. (Malraux, 1937: 201)

5 Cette réflexion pourrait ouvrir une perspective de recherche sur la constellation des symboles autour de la cécité permettant d'accéder à une lucidité supplémentaire ou à une imagination dépassant la surface sensible des yeux (à partir du début de la tradition littéraire occidentale, Homère). 
Dans ce moment même du récit, quand une œuvre d'art surgit, elle n'est pas décrite pour son contenu, mais pour sa surface touchée par les conséquences de la bataille, indiquée du doigt par un des personnages du livre, devenu actant, comme dans un tableau du Greco:

Le Négus montrait de son index court une crucifixion à la Bonnat, pâle sur un fond de bitume, fusillée depuis des jours par ceux d'en face. Les trous groupés des balles avaient presque arraché le bras droit ; le gauche, protégé sans doute par les pierres des murs, était seulement percé çà et là ; de l'épaule à la hanche, en bandoulière, le corps blême avait été parcouru par une rafale de mitrailleuse, régulière et nette comme la piqûre d'une machine à coudre. (Malraux, 1937: 199)

Il s'agit d'un double dispositif où les objets à côté des êtres humains deviennent des tableaux dont la temporalité est suspendue et les œuvres d'art entrent en contact avec les hommes par le biais des blessures que les hommes mêmes laissent dans les tableaux. Toujours dans les chapitres dédiés à Tolède, ce dispositif à double détente s'élargit de plus en plus à la ville toute entière, comme on peut l'apprécier dans plusieurs passages, par exemple celui de la fuite des citoyens avant l'invasion franquiste:

Derrière des groupes silencieux passaient des charrettes bosselées de paniers et de sacs, où brillait un instant l'éclat écarlate d'une bouteille ; puis, sur des ânes, des paysannes sans visage, et dont pourtant on devinait le regard fixe, avec la séculaire détresse des Fuites en Égypte. L'exode coulait, enfoui sous ses couvertures dans cette odeur de feu, scandé par le battement profond et rythmé du canon. (Malraux, 1937: 228)

Une expression telle que la terre semble s'être fendue quelque part dans sa profondeur, fait tomber en pluies feuilles et brindilles mortes. (Malraux, 1937: 232)

Toute la ville, enfin, est affectée par cette dynamique qui se répand comme l'odeur des cadavres et qui la transforme en une gigantesque nature morte, ou plutôt en un cadavre géant :

Quand retombait avec le vent le bruit du canon et des fusils, un autre bruit continuait, faible et poignant : le bruit crépitant, étouffé, des flammes du toit de l'Alcazar. Ce bruit-là s'accordait à l'odeur qui rendait dérisoire le canon, les appels éloignés, et tout ce qui venait de l'agitation des hommes : l'odeur de feu et de cadavres mêlées, si épaisse qu'il semblait que l'Alcazar n'y pût suffire, qu'elle ne pût être que l'odeur même du vent et de la nuit (Malraux, 1937: 220)

L'odeur de cadavres et de pierre chaude l'entoura, disparut une seconde dans le vent, remonta et reprit possession du jardin plein de manteau (Malraux, 1937: 222)

La ville se vidait comme un corps perd son sang (Malraux, 1937: 240)

Cet univers extrahumain et anthropomorphisé à la fois ressent donc du contact avec les manifestations les plus violentes du combat, mais parfois la réaction de la part de ces êtres apparemment inanimés est très mesurée, presque impalpable, comme dans le cas suivant, dans un autre passage du livre :

Le canon battait régulièrement comme le cœur de cette foule, au-dessus des minces coups de fusil qui partaient de toutes les fenêtres et de toutes les portes, au-dessus des cris, de l'odeur de pierre chaude et de bitume qui montait de Madrid. [...]

Dans les verres immobiles le cognac et la manzanilla tremblotaient au rythme du combat. (Malraux, 1937: 41-42)

Marcelino, aussi, se confronte au ciel et à l'indifférence des planètes, peu avant de mourir: 
Ils retrouvèrent le ciel, immobile au-dessus des nuages qui semblaient suivre le mouvement de la terre. $[\ldots]$

Jaime, mitrailleur-avant, fit un signe à Marcelino : pour la première fois, tous deux prenaient conscience dans leur corps du mouvement de la terre. L'avion qui tournait, comme une minuscule planète, perdu dans l'indifférente gravitation des mondes, attendait que passât sous lui Tolède, son Alcazar rebelle et ses assiégeants, entraînés dans le rythme absurde des choses terrestres. (Malraux, 1937: 146)

Ces descriptions associent les objets, les œuvres d'art et les hommes, mais elles marquent néanmoins cette indifférence des objets face à l'agitation des êtres humains. Pourquoi cette présence si insistée de telles descriptions?

Une première hypothèse de réponse est qu'elles préfigurent la destinée des actions humaines, qui devront tôt ou tard abandonner leur rythme effréné et rejoindre celui des natures mortes. Mais dans cette présence, il y a aussi l'idée que les natures mortes et la présence des animaux représentent des «still lifes», des vies suspendues et silencieuses : la mort des hommes devient, grâce à cette présence préalable des éléments extrahumains dans l'expression artistique, une suspension de la chute pour faire entrer les corps humains dans la dimension atemporelle, où l'acte de tomber est détourné de sa tendance à la chute, pour ensuite suspendre cette même descente et afin que les hommes puissent finalement tomber vers le haut, comme Simone Weil le propose.

Malraux semble développer ailleurs cette hypothèse, dans L'Intemporel, quand il associe certaines Natures mortes de Cotan, de Chardin ou de Vermeer à la naissance de la photographie:

L'opération créatrice, dans les civilisations historiques, consiste à arracher ce qu'elle représente, au monde de l'apparence, de la mort et du destin, pour l'annexer à un monde dont notre civilisation connaît pour la première fois la totalité, et que nous appelons le monde de l'art. [...]

La photographie, la peinture qu' elle suscite montrent à merveille que l'art a toujours créé une autre corrélation que celle de la vie ; la corrélation des formes de la vie n'a pas d'histoire, même si l'humanité en a une. (Malraux, 1976 :725)

La transition de la chute des hommes vers une suspension devenant une élévation est dans la partie de L'espoir consacrée à la chute de Tolède dans les mains des rebelles franquistes, notamment dans le chapitre consacré à l'exécution des républicains, triés en lignes de trois et fusillés (dans le chapitre 10, sous-section II, section II «Exercice de l'Apocalypse, de la Première Partie). Malraux choisit de voir cette scène par les yeux d'un des prisonniers, Hernandez:

Tolède rayonne dans l'air lumineux qui tremble au ras des monts du Tage : Hernandez est en train d'apprendre de quoi se fait l'histoire. Une fois de plus, dans ce pays de femmes en noir, se lève le peuple millénaire des veuves. [...] Les trois prisonniers sont enfin de face : la photo est décidément prête.[...]

Décharge. Deux tombent dans la fosse, un en avant.[...]

Trois nouvelles silhouettes sont debout là où se sont trouvés toutes les autres.

(Malraux, 1937: 256-257) 
Mais c'est encore là que Malraux fait intervenir un mouvement du regard ou un changement de focalisation, car il se détache du point de vue d'Hernandez, pour clôturer le chapitre et la chute de Tolède par son propre regard sur Hernandez qui meurt, qui tombe et qui monte par sa chute:

Trois autres, dont Hernandez, montent, dans l'odeur d'acier chaud et de terre remuée.

(Malraux, 1937: 257)

La ville et les corps rayonnent et montent par le sacrifice, au lieu de tomber, au bout de ce parcours qui transforme la ville en nature morte pour la faire entrer en contact avec une atemporalité dépassant la chronique, une atemporalité qui nivelle les chutes et les élévations dans un parcours indéchiffrable, qui échappe à la verticalité humaine.

Dans le film, le chemin des natures mortes est présent dès la quatrième scène, quand l'action se déplace du terrain d'aviation à une ville, qui pourrait être Barcelone, car les événements qui suivent dans les films font référence au deuxième chapitre de la première sous-section de «L'illusion lyrique», qui se passe à Barcelone et qui relate de l'action de Puig pour détruire le canon.

Malraux fait précéder l'acte de Puig (devenu Carral dans le film) par une réunion dans une arrière-boutique de la ville sous le bombardement. Les bruits des coups du canon occupent les images de la ville vue de l'extérieur et se transmettent aussi dans l'intérieur de l'arrière-boutique, mais le passage de l'extérieur à l'intérieur nous intéresse car, si l'image en extérieur (le plan 33) ne ressent pas du bombardement au niveau visuel (si on coupe le son, l'on dirait une image d'une ville dans un moment quelconque de sa vie), au contraire l'image à l'intérieur de l'arrière-boutique (le plan 35) transforme le bombardement sonore en une micro-chute visuelle se passant dans une nature morte en miniature, renfermé dans une petite boîte à papillons, qui est suspendue sur un des murs de la salle.

On assiste ainsi à un dédoublement du processus de la chute décrit avant pour le roman: d'un côté on a une référence évidente à des tableaux des natures mortes tels que L'Europe ou L'Asie de Jan van Kessel (Les quatre parties mondiales, huile sur cuivre, 1664), de l'autre l'on assiste à une chute physique à l'intérieur de la disposition ordonnée des papillons dans la nature morte. Tout cela est complété par le fait que les références picturales qu'évoque ici Malraux ne sont que des natures mortes qui ont comme décor un lieu associable à un musée, voire qui représentent des collections d'un musée dédié aux Continents de la terre.

Mais la question ne s'arrête pas là. Malraux lie directement cette petite chute en vertical à un mouvement dans la profondeur du plan, parce qu'à la chute des papillons un travelling en arrière va suivre, replaçant tous les éléments dans un autre contexte, celui de la réunion et du dialogue entre Carral et les autres républicains, comme si l'enchaînement entre la chute sonore des bombes se transférait dans cette énergie visuelle de la nature morte (miniature du musée de Tolède dans le livre?) et de la chute qui met du désordre dans l'ordre atemporel de la nature morte même. 
Une nécessité figurative semble régir cet enchaînement aboutissant dans une chute en horizontal de la profondeur de champ et liant la chute des papillons au personnage de Carral. Cette nécessité figurative semble s'amplifier ensuite dans la destinée de Carral qui décide de se lancer avec une voiture, comme un projectile, sur le canon qui inonde de son bruit la ville et qui a causé la chute des papillons.

Mais revenons au dialogue dans la boutique: la chute des papillons trouve une rime dans la fin de la même scène, avec le travelling latéral sur le bocaux, où des gouttes d'eau tombent, tandis que les hommes s'affairent dans le fond du plan. La chute des papillons et le travelling en arrière évolueraient donc vers un mouvement composé par le travelling latéral et par la chute des gouttes, comme dans un sablier, faisant appel à d'autres souvenirs de natures mortes. Mais les renvois d'une chute à l'autre, et d'un mouvement à l'autre - qu'ils soient un travelling ou un mouvement des corps humains ou extrahumains - ne s'arrêtent pas là.

Les deux travellings peuvent être considérés comme les déclencheurs de ce qui va suivre dans les plans de la voiture lancée contre le canon (les plans allant du 106 au 130): l'avancée horizontale de l'automobile remplace et renverse le mouvement du travelling en arrière, jusqu'au contact avec l'entité corporelle du canon. L'entrechoquement entre l'immobilité de la masse du canon et la force dynamique horizontale de la voiture semble précipiter l'arrêt forcé de cette dernière dans un triple envol: celui de la voiture qui carambole sur le canon, celui du corps de Carral qui s'envole pour tomber ensuite à côté de la voiture et l'envol collectif des oiseaux qui finalement synthétisent toutes les chutes précédentes dans un cycle commencé par les ailes des papillons et se terminant dans les ailes des oiseaux. Carral accomplit donc la relation entre l'atemporalité de la nature morte atteinte par la micro-chute et l'atemporalité de l'envol des oiseaux, et transforme en même temps la chute des papillons en un mouvement composé de son mouvement descendant et de celui, ascendant, des volatiles.

Le ciel où les oiseaux s'envolent, est le même ciel qui assiste à la chute de l'avion de Marcelino, et le même qui assistera au choc final de l'avion sur la montagne: la nature morte de la boîte aux papillons, dans sa chute en miniature, affecte l'expression visuelle de tout mouvement humain, pendant que la ville du film devient comme Tolède, un corps mort pour le sacrifice, qui trouve son incarnation et sa sublimation dans le corps déchu de Carral, dont la force morale devient la force visuelle du mouvement des oiseaux $x^{6}$.

6 Il faut considérer comment Malraux condense ici les deux chutes successives de Puig dans le roman: À peine dix minutes. Les soldats affolés se retournèrent, les artilleurs essayèrent de tourner leurs pièces : l'auto de Puig, le petit poste de garde enfoncé, dégringolait sur les canons avec le fusilmitrailleur entre deux lames du pare-brise, l'arrière secoué de gauche à droite comme un balancier frénétique. Puig voyait les canonniers, que leurs pare-balles ne protégeaient plus, grossir comme au cinéma. Une mitrailleuse fasciste tirait et grossissait. Quatre trous ronds dans le triplex. Penché en avant, exaspéré par ses jambes courtes, Puig écrasa l'accélérateur comme s'il eût voulu enfoncer le plancher de l'auto pour atteindre ses copains de l'autre côté des canons. Deux trous de plus dans le triplex, givrés. Une crampe au pied gauche, les mains crispées sur le volant, des canons de mousquetons qui se jettent sur le pare-brise, le fracas du fusil-mitrailleur dans les oreilles, les maisons et les arbres qui basculent, - le vol des pigeons juste en train de changer de couleur en même temps que de direction, - la voix du Négus qui crie... 
La complexité de ce stade de la recherche expressive sur la chute et la nature morte se propage aussi ailleurs. Il faut remarquer la présence d'autres entités qui pourraient enrichir cette hypothèse sur les natures mortes: dans un plan de la voiture de Carral allant vers la mort, il y a un chien (plan 111) qui n'est pas présent dans les plans de la voiture garée. On peut le considérer comme une méprise ou une faute de raccord de la part de l'équipe de Malraux, mais sa trace reste au montage, tout comme la trace d'un autre élément du monde animal, à la fin du film, quand le bombardier Schreiner (autre figure de la chute qui demanderait un parcours à part dans cette étude) est en train de tirer contre un chasseur ennemi : il voit une fourmi se baladant sur son viseur (plan 488). Une autre faute de raccord, une autre erreur, avec la présence improbable de cet être si petit sur un avion, mais cela est intéressant car cette présence miniaturisée par rapport aux hommes précède l'entrechoquement de l'avion de Schreiner contre la montagne, causant la scène finale $\mathrm{du}$ film et renvoyant l'avion dans l'ordre des objets minuscules face au gigantisme de l'élément naturel. Il y aurait par conséquence la coprésence de toutes les échelles possibles des êtres naturels et les relations entre $l^{\prime}$ homme et son monde se renverseraient dans un cycle visuel, où le regard de l'homme se trouve tiraillé entre l'extrêmement petit et l'extrêmement grand et sa chute ne serait qu'un choc mettant en contact les micro-chutes avec la macro-chute humaine ${ }^{7}$.

Une autre trace de ces passages entre le monde animal et les natures mortes est dans le plan nocturne de la scène où Peña cherche les voitures nécessaires à signaler la limites du terrain d'aviation pour l'envol des deux avions allant accomplir la mission à la fin du film (plan 268): Peña est porteur d'un regard s'étalant dans la profondeur de champ, sur des paysans transportant des quartiers de bœuf sur leurs dos, référence visuelle évidente aux tableaux de Rembrandt et de Carrache ${ }^{8}$. Il y a également la mort du guide du paysan, tué par le propriétaire d'un bistrot, qui va être tué à son tour par

Puig sortit de l'évanouissement pour retrouver la révolution et les canons pris. Il n'avait reçu qu'un choc très fort à la nuque quand l'auto avait basculé. Deux de ses compagnons étaient tués. Le Négus le pansait. (Malraux, $1937: 32$ )

Puig ne meurt donc pas du choc avec le canon, mais dans un autre combat à la fin du même chapitre. Le film et ses images permettraient donc à Malraux de synthétiser dans un mouvement à la double puissance ce qui dans le livre était divisé en deux mouvements.

7 D'où provient cette double vision entre l'extrêmement petit et l'infiniment grand? Une des sources de cette écriture de l'auteur de L'espoir pourrait être la description de la condition humaine telle qu' exprime Pascal dans les Pensées, surtout le fragment 185 (édition Lafuma):

Car enfin qu'est-ce que l'homme dans la nature? Un néant à l'égard de l'infini, un tout à l'égard du néant, un milieu entre rien et tout, infiniment éloigné de comprendre les extrêmes; la fin des choses et leurs principes sont pour lui invinciblement cachés dans un secret impénétrable. (Pascal, 1995: 153-160)

Dans ces pages, Pascal concentre exactement cette question de la vue de l'homme qui va et vient entre les abîmes du minuscule et ceux de l'immensité de l'univers, pour souligner enfin les limites de la connaissance humaine face à ce qui au-delà du spectre de vision de l'œil anthropocentrique.

8 Les références picturales sont multiples et peuvent être consultés dans le livre de Schreider sur les natures mortes: Rembrandt, Le Bœuf écorché ; Annibal Carrache, La boucherie,1580; Jan Van Kessel, L'Europe (panneau central), 1664-66, Huile sur cuivre, Munich, Alte Piankothek, L'Asie, (Schneider 2003 :162-165); pour ce qui concerne la présence des verres et des bocaux : Pieter Claesz, Nature morte, 1633, Huile sur bois de chêne, Kassel (Schneider 2003: 21); Entourage de Massys, Jérôme, vers 1520, Huile sur bois, Düsseldorf (Schneider 2003: 81), Georg Flegel (1566-1638), Nature morte avec pain et sucreries, non daté, Huile sur bois, Francfort, Georg Flegel, Nature morte de desserts, non daté, Huile sur bois, 
le paysan même (plans 219-220). Cette double chute, d'un républicain et d'un traître, va se condenser dans le plan de la fleur, après la chute du franquiste : ce plan tourné en contre-plongée, comme si le point de vue pouvait correspondre à celui du propriétaire mort et qui ne peut plus voir, indique comment ces réseaux de regards impossibles et de présences extrahumaines improbables ne peut être fondé que sur une instance de regard externe, prenant en compte la mesure du temps de la guerre et, en même temps, l'éternité cyclique des êtres naturels qui assistent aux éphémères actes humains.

L'image qui se charge de tous ces regards impossibles, de tous ces liens entre l'humain et le non humain, l'image qui exprime tous ces signes pourrait indiquer que la seule possibilité de contact entre les différentes dimensions du temps (celui des hommes, celui des autres êtres) peut passer par la chute, articulée sous les formes horizontales ou verticales, descendante ou ascendante, mais en tout cas par un mouvement similaire à celui d'une précipitation chimique des êtres les uns vers les autres, en passant par des réminiscences des œuvres d'art : cette précipitation permettrait le contact avec les deux temporalités, mais également le contact de l'homme avec son contraire, avec son ennemi, dans une annulation des mouvements contraires exprimée par la résultante des envols, un point de vue en contre-plongée, comme dans le plan des oiseaux après la mort de Carral et dans le plan la fleur dans le cas de la mort du guide et de celui qui l'a tué.

Finalement cette synthèse des mouvements opposés exprimerait-elle la grâce morale de certains corps contre la mort des ennemis? La question demeure ouverte surtout parce que ce qui reste est justement cette réunion des êtres dans le même espace, êtres ennemis morts ou vivants, temporaires face au regard d'une nature suspendue par un point de vue différent par rapport à celui des hommes. Ce qui semble ressortir du travail figuratif de Malraux dans le film par rapport au travail imaginatif de l'écriture est le passage obligé de la chute humaine à travers la médiation de la réminiscence visuelle de la nature suspendue, morte ou plutôt qui regarde éternellement les générations des hommes qui tombent sur sa surface.

\section{LA DÉPOSITION MULTIPLIEE, LE REGARD ET LA DIAGONALE.}

Ce passage de la chute par la reconnaissance de soi-même dans le patrimoine artistique et éternel se condense à la fin du film dans la descente des corps des aviateurs dont l'avion s'est écrasé contre une montagne: tous les gestes descendants se résument et se retrouvent dans ce sommet de l'acte de tomber, et les deux mouvances détectées dans la mort de Marcelino et dans les natures mortes semblent aboutir à une troisième solution expressive de la chute.

Dans la partie finale du livre, deux avions sont chargés d'accomplir une mission, mais l'un d'entre eux, après un rude combat, s'écrase contre une montagne. Nous ne nous occuperons ici de la scène du choc de l'avion contre la montagne, parce que cet

Munich, Georg Flegel, Grand Repas de spectacle, non date, Huile sur cuivre, Munich (Schneider 2003: 88, 91, 93, 97); Willem Claesz, Heda Nature morte, 1651, Huile sur bois, Vaduz (Schneider 2003: 100). 
entrechoquement est une amplification de ce que nous avons déjà examiné dans le choc de Carral. Ce qui nous intéresse est la suite de ce choc, avec une chute ultérieure, à savoir l'acte descendant des aviateurs blessés qui sont transportés par la foule des paysans dans la vallée, en bas.

Cette descente va se transformer dans le livre en une élévation, grâce aussi à la force picturale que le cortège dans la vallée évoque à la mémoire des hommes. La descente vers le haut commence par celui qui a été blessé horriblement sur la partie centrale de son visage, qui arrive à peine à regarder, le personnage de Gardet:

Des paysans, en effet, venaient vers eux, amenés par celui qui s'était enfui lorsqu'il avait vu Gardet.[...]

Les paysans commencèrent à courir. Sans doute avaient-ils supposé que les aviateurs tombés étaient des leurs, car ils arrivaient presque sans armes [...]. Gardet vit le miroir du rétroviseur suspendu à sa place dans le fouillis de poutrelles et de fils, devant le siège de Pujol. «Si je me regarde, je me tue.» (Malraux, 1937: 467)

Puis tout de suite le regard passe de Gardet aux paysans:

Quand les paysans avaient été assez près pour voir le fatras d'acier hérissé de plans et de morceaux d'ailes, les moteurs défoncés, une hélice pliée comme un bras et les corps allongés sur la neige, ils s'étaient arrêtés. Gardet venait vers eux. [...]

Ils étaient revenus vers les blessés. Dès que les paysans eurent compris qu'un seul des allongés était mort, commença une agitation affectueuse et maladroite. (Malraux, 1937: 467)

Ensuite la focalisation interne et le regard reviennent à Gardet, qui ne voit presque pas (comme Schreiner tombé avec l'avion, auparavant dans le film):

Transporter Scali, Mireaux, le bombardier, ça ne s'annonçait pas simple; mais les montagnards ont l'habitude des jambes cassées. Pujol et Langlois pouvaient marcher. Et lui-même à la rigueur.

Ils avaient commencé à descendre vers le petit village, hommes et femmes tous petits sur la neige. Avant de s'évanouir, Gardet avait regardé une dernière fois le rétroviseur; il avait été pulvérisé dans la chute : il n'y avait jamais eu de miroir dans les débris. (Malraux, 1937: 468)

Le regard passe maintenant de Gardet à Magnin venu au secours des blessés, un regard qui porte la description des visages des blessés transfigurés après la chute, comme si cette chute avait accéléré le temps de la transformation des linéaments :

La première civière débouchait en face de Magnin. Quatre paysans la portaient, chacun un brancard sur l'épaule, suivis aussitôt de quatre camarades. C'était le bombardier.

Il ne semblait pas avoir la jambe cassée, mais des années de tuberculose. La face s'était durement creusée, donnant aux yeux toute leur intensité, et changeant en masque romantique cette tête à petites moustaches de fantassin trapu.

Celle de Mireaux, qui suivait, n'avait pas moins changé, mais autrement : là, la douleur était allée chercher l'enfance.

- On est partis de là-haut dans la neige qui tombait! dit-il quand Magnin lui serra la main; c'est marrant! il sourit et referma les yeux.

Magnin continua d'avancer, les porteurs de Linares derrière lui. La civière suivant était assurément celle de Gardet: un pansement recouvrait le visage presque tout entier. Seule chair de tout le corps, paraissaient les paupières gonflées à éclater, mauve pâle, serrées par l'enflure l'une contre l'autre, entre le serre-tête et le pansement plat qu'il maintenait, et sous lequel le 
nez semblait avoir disparu. Les deux premiers porteurs, voyant que Magnin voulait parler, déposèrent la civière avant les seconds et, pendant un instant, le corps demeura oblique, comme une Présentation du combat. (Malraux, 1937: 468)

Geste inattendu où le corps retrouve une verticalité inattendue, que Malraux reprend dans le film, et que nous retrouverons dans une autre image, celle du vieillard malade, transporté dans un lit disposé en vertical dans les escaliers d'un immeuble romain, dans Umberto D. (1952) de De Sica.

Visage sans nez, tout comme le lépreux que François d'Assise serre dans ses bras dans Les onze fioretti (1950) de Rossellini, où la douleur et la grâce se retrouvent dans l'arrêt que François impose au chemin du souffrant pour arriver au contact, tout comme Magnin ici impose un arrêt pour se rapprocher de ce qui reste du visage de Gardet. Désormais les mouvements du corps de Gardet sont délégués aux hommes qui le transportent, seul le regard brouillé lui reste, mais Magnin ne le sait pas encore, il pense que le regard aussi n'existe plus:

Aucun geste n'était possible : les deux mains de Gardet étaient sous la couverture. Entre les paupières de gauche, Magnin crut entrevoir une ligne :

- Tu vois?

- Pas trop. Enfin, je te vois, quoi !

Magnin avait envie de l'étreindre, de le secouer. (Malraux, 1937 :468)

L'espoir ici passe donc par une vue qui devient la source même d'une force cinétique de contact, de vie enfin, qui semble se transmettre de Magnin à la civière et au peuple derrière elle, conscient de la douleur profonde de cet homme sans plus visage: «La civière se remit en marche, la moitié de Valdelinares derrière elle.». Après cette impulsion de contact, Magnin désormais laisse couler le mouvement de descente des civières et de la foule, comme si son mouvement ne pouvait plus arrêter ni retenir cette descente, mais comme s'il la multipliait :

Magnin laissa passer devant lui civières et paysans jusqu'aux derniers, qui portaient le cercueil ; il avait été fait plus vite que les civières: l'habitude... Sur le couvercle, les paysans avaient attaché une des mitrailleuses tordues de l'avion.

Toutes les cinq minutes, les porteurs se relayaient mais sans poser les civières. (Malraux, 1937: 469)

$C^{\prime}$ est dans ce mouvement de descente qui ne peut, qui ne doit pas s'interrompre pour pouvoir devenir ascension à la deuxième puissance, qui surgit la figure d'une femme:

- Quel âge a-t-il ? dit-elle en montrant Mireaux.

- Vingt-sept ans.

Depuis quelques minutes, elle suivait la civière, avec le désir brouillon d'être utile, mais aussi avec une tendresse délicate et précise de gestes, une façon de caler les épaules chaque fois que les porteurs, dans une descente très raide, devaient assurer leurs pieds, où Magnin reconnaissait l'éternelle maternité.

La vallée descendait de plus en plus. D'un côté, les neiges montaient jusqu'au ciel sans couleur et sans heure; de l'autre, de mornes nuages glissaient au-dessus des crêtes. (Malraux, 1937: 469) 
Et encore, à la fin, la descente est une élévation, une traversée de la montagne au contraire, avec les mouvements qui se transfèrent des blessés aux transporteurs, des hommes aux femmes et à la terre et au ciel entier:

Magnin, presque à la fin du cortège, revint jusqu'à la civière de Scali. C'était le seul qui pût s'accouder: devant lui, le sentier descendait en zigzags presque égaux jusqu'à Langlois, arrêté devant un mince torrent gelé. [...] Les brancards, l'un après l'autre, passaient le torrent : le cortège, de profil, se déployait sur l'immense pan de roc aux ombres verticales.

-Voyez-vous, dit Scali, j'ai eu autrefois....

- Regarde ça: quel tableau!

Scali rentra sont histoire; sans doute eût-elle tapé sur les nerfs que Magnin, comme la comparaison d'un tableau et de ce qu'ils voyaient tapait sur les nerfs de Scali.[...] Son brancard passa le torrent. En face reparurent les taureaux. Espagne de son adolescence, amour, décor et misère! L'Espagne c'était cette mitrailleuse tordue sur un cercueil d'Arabe, et ces oiseaux transis qui criaient dans les gorges. (Malraux, 1937: 470-471)

Encore une fois l'élément visuel et le regard vont réunir le mouvement descendant des corps humains avec la vision ascendante des oiseaux. Cette présence deviendra de plus en plus insistante du point de vue auditif dans l'avancée du texte, tout en signalant l'éloignement visuel des oiseaux. Leur son est identifié à ceux des rapaces, qui viennent scander comme une rime chaque fin de description du cortège, comme ans les extraits suivants:

Pas d'averse: c'était le bruit des torrents dont le rocher l'avait séparé, ainsi que d'un perspective, et qu'on n'entendait pas de l'autre versant [...]. Le soir ne venait pas encore, mais la lumière perdait de sa force. Magnin, statue équestre de travers sur son mulet sans selle, regardait le pommier debout au centre de ses pommes mortes. [...] Dans ce silence empli tout à coup de ce bruissement d'eau vivante, cet anneau pourrissant et plein de germes semblait être, au-delà de la vie et de la mort des hommes, le rythme de la vie et de la mort de la terre. Le regard de Magnin errait du tronc aux gorges sans âge. L'une après l'autre, les civières passaient. (Malraux, 1937: 471)

Sans qu'il comprît trop bien comment, la profondeur des gorges où ils s'enfonçaient comme dans la terre même, s'accordait à l'éternité des arbres. Il pensa aux carrières où on laissait jadis mourir les prisonniers. Mais cette jambe en morceaux mal attachés par les muscles, ce bras pendant, ce visage arraché, cette mitrailleuse sur un cercueil, tous ces risques consentis ; la marche solennelle et primitive de ces brancards, tout cela était aussi impérieux que ces rocs blafards qui tombaient du ciel lourd, que l'éternité des pommes éparses sur la terre. De nouveau, tout près du ciel, des rapaces crièrent. (Malraux, 1937: 472)

Derrière les créneaux tout Linares était massé. [...]

Tous les autres blessés voyaient ; et quand ils avaient vu la foule, ils s'étaient efforcés de sourire, même le bombardier. Gardet ne regardait pas. Il était vivant : des remparts, la foule distinguait, derrière lui, le cercueil épais. Recouvert jusqu'au menton par la couverture, et, sous le serre-tête en casque, ce pansement si plat qu'il ne pouvait y avoir de nez dessous, ce blessé-là était l'image même que, depuis des siècles, les paysans se faisaient de la guerre. [...] Les femmes pleuraient sans un geste, et le cortège semblait fuir l'étrange silence des montagnes, avec son bruit de sabots, entre l'éternel cri des rapaces et ce bruit clandestin de sanglots. (Malraux, 1937: 474)

Cette fin du cortège identifie entre deux sons toute la démarche paradoxale entre l'éternité et l'éphémère clandestinité des hommes, en faisant déborder le «trop plein» visuel de cette descente dans la coexistence des deux sons, tandis que les images du pommier et des corps, multitude de dépositions répétées, multitude d'images de 
sacrifice et de guerre, reviennent aux hommes pour rappeler les images des siècles, les images déposées dans la mémoire collective, pour reconnaître l'image du blessé et de la guerre. Ce processus de réminiscence va brasser donc cet ensemble des images pour arriver au contact entre le haut du cri éternel des rapaces et le bas du sanglot temporaire des femmes: ce contact exprimerait l'aboutissement de cette chute vers le haut qui est le sacrifice des hommes devenu symphonie temporelle des sons.

Ce qui nous interpelle est finalement la transformation de tout cela dans la fin $\mathrm{du}$ film. On peut distinguer trois niveaux de questionnement dans l'adaptation de ces figures de la verticalité dans le film: tout d'abord une évidente construction du cadrage à partir, encore une fois, du dispositif de la réminiscence des œuvres d'art de la tradition chrétienne; ensuite la place du regard humain se relayant avec l'espace naturel, avec l'envol des oiseaux qui viennent remplacer les sons des rapaces dans le livre; finalement la mise en place d'une rhétorique de la représentation du peuple par l'intermédiaire d'une disposition diagonale de la foule en profondeur dans la construction des plans, avec des références à la tradition cinématographique précédente et des liens évidents avec d'autres œuvres cinématographiques concentrées autour de la question politique et du combat contre le fascisme.

La réminiscence de plusieurs images de la Déposition et de la Descente de la Croix habitent les images qui se concentrent autour du personnage de Marquez, le corrélatif filmique du Gardet littéraire : l'évidence du travail de la composition du plan de la part de Malraux est démontrée dès qu'on compare ces plans aux tableaux de Caravage, de Rembrandt et de Pedro Machuca. Le travail de Malraux touche aussi la direction des acteurs, quand il dispose le bras de Marquez et d'un autre blessé comme dans les tableaux en questions : il y a donc une volonté d'identification entre cette image filmique et la tradition figurative concernant l'image-catalyseur de la Déposition, symbole émotionnel de descente et d'ascension simultanées.

Mais un autre choix créatif du réalisateur est exprimé aussi dans les images qui montrent la partie du livre où les paysans qui transportent Gardet sur la civière s'arrêtent et disposent le corps de l'aviateur presque en vertical, un peu de biais. Nous ne savons pas si Malraux avait à l'esprit les images de Mantegna du supplice de Saint Sébastien ou de la mort du Christ quand il a écrit ce passage du livre, ce qui est certain est le choix de cadrage, tout comme le choix de disposer le corps de Marquez-Gardet à mi-chemin entre la posture horizontale du martyre et la posture horizontale de la célèbre Lamentation du Mantegna, sublimation de la perspective en fonction émotionnelle, qui habite tous les plans de ce cortège avec les civières des blessés avançant en horizontal.

Une partie du récit ajouté au tournage et absente du roman pose davantage de problèmes. Jusqu'à maintenant le regard sur les blessés a été confié à PeñaMagnin, comme il est dit dans le texte pour la majeure partie. Mais, précisément, dans le film, Marquez rappelle Peña pour lui demander de lui donner un miroir, car il veut se regarder. Le commandant trouve un miroir mais il ment au blessé, pour d'évidentes raisons d'humanité. Pourquoi alors Malraux introduit-il cette pause, voire 
ce renversement dans le chemin à rebours de Peña, en train de monter pendant que les autres descendent ? Malraux se sert de ce mécanisme du miroir pour harmoniser les deux chemins, celui du commandant et celui du cortège, comme si seulement cette harmonie pouvait permettre l'avancement de la chute vers le haut. Pour ce faire, il a besoin d'un basculement du regard entre Peña et Marquez, jusqu'à ce que Peña récupère la maîtrise du regard sur les blessés. La tentative de transmission du regard du commandant à un des blessés a déclenché une étincelle ou une possibilité de délégation de la vision subjective de cette descente, qui aboutit immédiatement dans l'attitude de Schreiner.

Il faut se souvenir que Schreiner a un rôle important dans le film, beaucoup plus que dans le livre: il est celui qui tombe avec son avion pendant un exercice sur le terrain de l'aviation et qui est atteint aux yeux à cause de cette chute. Mais cet ancien pilote allemand, qui a passé des années dans les mines, décide quand même de se rendre utile au combat en devenant bombardier. À la fin du film, il est gravement blessé et sait qu'il est en train de mourir. C'est pour lui que Peña descendra de son âne, en accomplissant la synthèse de tous les gestes de descente qui aboutiront à la grâce morale dans l'arrivée au village en bas. Mais cela s'accompagne par la seule délégation du regard que Peña concède au blessés, le seul moment où le commandant cède la maîtrise du regard à cet aviateur dont la vue est abîmée, tout comme la vie. Schreiner, après avoir parlé à Peña, lève les yeux au ciel et regarde, le plan qui suit est celui de la cible de son regard : des oiseaux qui s'envolent comme dans le moment où Carral tombe en s'envolant. L'esprit de Schreiner, ou sa grâce morale, semble s'envoler par le regard sur les oiseaux. Mais ce plan-là est aussi un procédé d'euphémisation: le son des rapaces du livre se transforme en envol de liberté de l'esprit en train de se détacher du corps.

Ces deux chemins de modelage de la chute semblent se compléter par le contact ultime entre les combattants et les paysans, surtout dans la forme de la diagonale que Malraux emploie de façon si insistante dans les mouvements collectifs de la descente vers le village. Nous entendons dire que Malraux et Max Aub ont organisé ces mouvements de milliers de corps humains qui amplifient tous les choix précédents relatifs à cette idée de la chute vers le haut par le modelage d'une diagonale sillonnant le plan et traversant la montagne, en sorte qu'une écriture de la descente semble être composée sur la surface même de la terre, notamment dans les derniers plans, où la puissance de ce cortège humain lie de manière indissoluble l'idée de la grâce par la descente à sa forme visuelle la plus évidente.

Si jusqu'à maintenant les suggestions imaginaires s'étendaient à une réflexion sur les actions humaines par rapport à la verticalité et aux références picturales, en revanche la recherche formelle dans cette partie finale du film trouve des chemins obliques entre l'horizon et la verticalité, avec un jeu de miroir par rapport à des films de l'encore jeune tradition cinématographique, ou en synchronie avec des films qui sont en train de naître ailleurs ou en Espagne même et qui se concentrent toujours autour de la question de la représentation d'un mouvement collectif d'élévation, de sublimation. 
Le plan 572, ainsi que tous les plans de la descente vers le village construisent une série de lignes de fuites dans la profondeur de champ, en explorant toutes les possibles issues diagonales offertes par le mouvement humain par rapport à la surface contenue dans le cadre, et en opposant parfois la verticalité des corps, notamment avec les bras levés, à ces lignes diagonales.

Le cas plus évident de référence cinématographique pour ce travail de l'écrivain et réalisateur français est la traversée des Alpes d'Annibal dans le long-métrage italien Cabiria (1913-14) de Giovanni Pastrone : la descente de nos paysans espagnols reprend cet acte de traverser le sommet des montagnes pour descendre et envahir les vallées dans un acte de conquête figurative. Il n'y a pas, bien sûr, aucune épreuve certaine de l'influence du film italien sur le film de Malraux, mais il y a cette coïncidence des intentions des deux réalisateurs dans l'utilisation des diagonales et de la profondeur dans l'acte de la descente devenant un acte de conquête qui nous pousse à interroger la valeur de ces moyens d'expression visuelle dans le sens d'un renversement du sens de la chute.

Ensuite, il y a le travail de modelage de la profondeur et de lignes diagonales tracés par les chemins du peuple dans la fin de la première partie d'Ivan le Terrible, réalisé en 1945 par Serguei M. Eisenstein : le témoignage de Denis Marion relate qu'Eisenstein était en contact avec Malraux depuis 1934, dont il aurait voulu adapter La condition humaine, avant l'engagement de l'écrivain dans la guerre en Espagne (Marion, 1970: 10). Une fois de plus, aucune épreuve n'est certaine d'une influence quelconque entre les deux films, d'autant plus que les copies de Sierra de Teruel étaient presque introuvables pendant la deuxième guerre mondiale, mais ce qui nous frappe est la convergence des moyens des deux artistes dans le modelage de la même tension corporelle d'un collectif d'êtres humains. Il y a, certes, des différences, notamment dans la centralité du personnage d'Ivan dans le film d'Eisenstein, tandis que les combattants du film de Malraux sont en symbiose, sont accueillis et forment un seul corps avec les paysans. Ce qui compte est le recours et le perfectionnement figuratif et émotionnel de la même écriture du paysage grâce à la disposition des corps dans les diagonales sillonnant le plan cinématographique : le peuple russe s'agenouille devant Ivan en créant une sorte d'élan qui finalise les mouvements des courbes dans la profondeur, tout comme la descente du haut au bas dans le film de Malraux est l'aboutissement de ces courbes tracées sur les montagnes. Ces diagonales parfois curvilignes renversent le sens même de la verticalité dans un vertige abstrait, graphique, où les matières sont épurées de leur pesanteur pour devenir dessin, construction idéale d'un mouvement descendant à la deuxième puissance, transformant le sens de la descente dans une chute vers le haut.

La référence la plus proche par rapport au travail de Malraux est probablement représentée par le film Ispaniya, qu'Esther Shub tourne pour le public russe, en se servant d'un opérateur très indépendant, qui deviendra ensuite le réalisateur militant le plus connu au service de toute révolution du Vingtième Siècle, Roman Karmen. Le documentaire Roman Karmen, un cinéaste au service de la révolution a mis en évidence l'usage que Karmen a su faire des défilés en forme diagonale pour construire les 
émotions des mouvements collectifs dans ses films, et on peut supposer que Ispaniya ressent déjà de cette recherche formelle de la part de Karmen, presque dans la même période du tournage de Sierra de Teruel.

Les renvois entre les deux films sont impressionnants, car il y a aussi les mêmes figures de la Déposition du Christ de Giotto dans le film de Karmen, et le réseaux des signes visuels renvoyant d'un film à l'autre se multiplient à tel point qu'une étude spécifique serait nécessaire: dans Ispaniya on a pu déceler, par exemple, la présence d'un soldat blessé au visage, tout comme Marquez. La coïncidence la plus frappante est dans les plans que Karmen tourne à propos de la défense de Madrid, avec les républicains qui creusent les tranchées autour de la capitale pour la défendre des franquistes. Les plans de l'attente des républicains dans les tranchées sont une véritable écriture dans la terre, une incision se disposant sur les diagonales et parcourant la profondeur de champ, comme si l'acte de creuser la terre correspondait à l'acte de Malraux de tracer ses lignes noires du cortège sur la montagne. L'acte de creuser, tout comme l'acte de filmer ces chemins, montrent comment le fait d'entrer dans la terre, de laisser sa trace visuelle et matérielle dans le terrain deviennent un acte de creuser vers le haut, à la recherche de l'image correspondant au sacrifice matériel d'un peuple.

Une première conclusion de notre enquête pourrait se prolonger afin de savoir si le film de Malraux peut être considéré comme un acte de propagande, lors que le lien est évident avec le réalisateur Karmen qui fut parmi les inventeurs de la propagande audiovisuelle. La différence entre Karmen et Malraux est dans le fait que l'acte de Malraux prend en compte la défaite et la transforme en acte de sublimation, avec une lucidité et une honnêteté rare, tandis que le modelage de Karmen est orienté vers un dogme: le peuple a toujours gagné, même quand il a perdu. Pendant le tournage de son film, Malraux, conscient que la guerre était perdue, érige ce martyre visuel en traçant tous ces chemins qu'on a essayé de repérer ici, des parcours qui atteignent les idées de Simone Weil et qui les mettent en relation avec cette opération récurrente de la réminiscence, de la mémoire, comme si l'espoir n'était que la mémoire des chemins parcourus pour atteindre le sacrifice de la pesanteur morale.

\section{CONCLUSION}

Trop d'éléments n'ont pas pu être étudiés à fond ici, car le film et le livre sont pétris d'images et de signes qui restent à explorer, comme l'image de l'enfant qui joue à l'aviateur à la fin du film, comme la chute de l'avion de Schreiner dans le champ d'entraînement de l'aviation républicaine, anticipation de la chute finale, tout comme d'autres éléments qui rendent ce film et son rapport à l'œuvre littéraire un multiplicateur de questions quant aux images entre l'écriture et l'imaginaire.

Un autre élément qui reste à explorer est la relation entre ce film et l'œuvre de Joris Ivens (Terre d'Espagne, 1937) sur la même guerre, tout comme il serait nécessaire un examen approfondi du rapport entre Sierra de Teruel et les films de Roman Karmen. 
Mais notre objectif était de démontrer la centralité d'une esthétique de la chute exprimée par Malraux dans son œuvre littéraire et filmique, tout comme le niveau de complexité et de finesse de cette question, touchant à un patrimoine inépuisable d'images des arts, allant de la peinture au cinéma. Si notre analyse s'est portée davantage sur le film, cela se justifie par la nécessité d'approfondir cet exemple particulier d'autoadaptation. Notre intention n'était que celle de contribuer à un débat, ou à l'ouvrir, sur un corpus intertextuel se concentrant autour de l'acte de création de Malraux, mais qui s'étend aux possibles influences et confluences de la pensée visuelle, allant de Simone Weil à la cinématographie d'Eisenstein.

Au montage, Malraux interrompt la scène de la descente des corps des blessés de la montagne une seule fois, pour montrer les vieux paysans dans un village en bas qui veulent aller en haut, qui s'entêtent d'aller saluer les corps des aviateurs. Un jeune homme du comité local des républicains demande à quoi cela sert, il le demande au plus âgé, à celui qui probablement a vécu le plus, à celui qui a vu le plus de défaites parmi les paysans. La réponse provient du visage de cet homme (dans le plan 571), un visage dont les rides symbolisent aussi les sentiers montagneux vus par Malraux, un visage-renfermant tous les parcours de chute et d'élévation qu'une vie humaine peut avoir vécus à la fin de son chemin. Il dira: pour lui être reconnaissant. Un lent fondu enchaîné va lier ce visage de vieillard au chemin de la montagne, quand le cercueil avance en traçant la plus longue diagonale du film. Pendant ce fondu, le vieil homme devient une présence fantomatique, une ombre ou une projection qui fusionne avec le mort à qui il veut être reconnaissant, en lui disant, presque comme dans le chant d'une autre résistance, celle de la France Libre: «Ami, si tu tombes, un ami sort de l'ombre à ta place». Les mots de Nietzsche au début du chapitre Le cri de détresse dans Ainsi parlait Zarathoustra reviennent à l'esprit : deux hommes se retrouvent et chacun d'entre eux traverse son propre visage de sa main droite, avant de serrer la main de son ami ancien et retrouvé. Ils se serrent la main, pour se reconnaître (Nietzsche, 1996 :346).

On ne retrouve pas de trace de ce dialogue-là du vieil homme dans le roman et, pourtant, il a une origine. Dans le septième chapitre de la première Section («Être et faire») de la Deuxième Partie: à la fin d'un long dialogue entre Scali et le père de Jaime, un passionné d'art et de peinture, ce vieillard, comparé par Malraux même à un personnage des tableaux du Greco (Malraux, 1937: 311), cite un vers en français: «Que te sert, ô Priam, d'avoir vécu si vieux!»(Malraux, 1937: 320). Le fondu enchaîné du film serait en quelque sorte la réponse à l'interrogation de ce vers du livre: cela a servi à reconnaître, tout comme à renaître, ou à revivre.

La reconnaissance se confond ici avec la réminiscence tactile et visuelle à la fois: un homme à la fin de son existence a besoin de se retrouver, de retrouver l'image de son propre sacrifice par le biais du sacrifice fraternel de l'autre, à travers tout son patrimoine d'images de martyres et de dépositions et à travers toutes les blessures de son visage transférées sur la peau de la main des images. Ce vieil homme renferme peut-être le sens de la quête formelle et morale de Malraux, dans la confusion entre la reconnaissance et la réminiscence, entre l'acte de se reconnaître et de se souvenir de 
toutes les images déjà passées du sacrifice humain, pour arriver enfin à l'espoir de la liberté.

\section{BIBLIOGRAPHIE}

Alpers, Svetlana. (1990) : L'art de dépeindre - La peinture hollandaise au 17ème siècle, Paris: Gallimard.

Aumont, Jacques, Bergala, Alain, Marie, Michel, Vernet, Marc. (1994): L'esthétique du film, Paris: Nathan.

Aumont, Jacques, Marie, Michel. (1999): L'analyse des films, Paris: Nathan.

AA.VV., (Octobre 1989), L'Avant-scène Cinéma : Espoir/Sierra de Teruel, Paris, n³85.

Bellour, Raymond. (1979) : L'analyse du film, Paris: Albatros. Gaudreault, André. (1999) : Du littéraire au filmique, Montréal-Paris:Nota Bene - Armand Colin.

Malraux, André. (1937): L'espoir, Paris: Gallimard, Folio, 1963.

Malraux, André. (2004): Les voix du silence, in CEuvres Complètes, Tome IV (Écrits sur l'art, Tomes I), Paris: Gallimard.

Malraux, André. (2004) : L'intemporel, in CEuvres Complètes, Tome V (Écrits sur l'art, Tome II), Paris: Gallimard.

Marion, Denis. (1970): André Malraux, Paris: Seghers.

Nietzsche, Friedrich. (1996): Ainsi parlait Zarathoustra, Paris: Flammarion.

Pascal, Blaise. (1995): Pensées, Paris: Gallimard.

Schneider, Norbert. (2003): Les natures mortes, Cologne - Paris: Taschen.

Weil, Simone. (1947): La pesanteur et la grâce, Paris: Plon, 1999.

\section{FILMOGRAPHIE}

Barberis, Patrick, et Chapuis, Romain, Roman Karmen, un cinéaste au service de la révolution (2002, France, 90').

Eisenstein, Serguei M., Ivan le terrible (1945 - URSS, 95'+81').

Ivens, Ioris, Terre d'Espagne (1937 - Espagne, 52').

Malraux, André, Espoir- Sierra de Teruel (1938-1939, Espagne-France, 70'+3').

Pastrone, Giovanni, Cabiria (1913-1914 - Italie, 126').

Rossellini, Roberto, Les onze fioretti de François d'Assise (1950 - Italie, 75').

Shub, Esfir, Ispaniya - Espagne (1939 - URSS, Espagne, 85'), tourné par Roman L. Karmen. 


\section{ANNEXES}

Tableau synoptique - La structure du film (Espagne-France, 1938-1939, N\&B, $35 \mathrm{~mm}, 70 \mathrm{~min}+3 \mathrm{~min}$. Introduction de M. Schuman) ${ }^{9}$

\begin{tabular}{|c|c|c|c|}
\hline Minutage & $\begin{array}{l}\text { Plans et } \\
\text { transitions }\end{array}$ & Structure narrative du film & Réf. au livre \\
\hline $\begin{array}{l}0^{\prime} 00^{\prime \prime}- \\
1^{\prime} 19^{\prime \prime}\end{array}$ & & Génériques (10 titres) & \\
\hline \multirow[t]{2}{*}{$10^{\prime \prime \prime}$} & & 1. PREMIER SEGMENT & \\
\hline & 0 & $\begin{array}{l}\text { TITRE : « Pendant la guerre civile d'Espagne, } \\
\text { en } 1937 \text {, un appareil républicain, regagne } \\
\text { après un combat le terrain d'aviation où des } \\
\text { volontaires de tous les pays se sont réunis } \\
\text { pour lutter contre le fascisme.» }\end{array}$ & \\
\hline \multirow[t]{2}{*}{$1^{\prime} 24^{\prime \prime}$} & $1-10$ & 1.A La chute de l'avion & $\begin{array}{l}5^{\text {ème }} \text { Chapitre, sous- } \\
\text { section I, section I, de } \\
\text { la Deuxième Partie } \\
\text { (page 162) }\end{array}$ \\
\hline & $\mathrm{FE}^{10}$ & & \\
\hline $1^{\prime} 55^{\prime \prime}$ & $11-25$ & $\begin{array}{l}\text { 1.B Le deuil pour la mort de Marcelino : le } \\
\text { discours de Peña }\end{array}$ & $\begin{array}{l}\text { Ibid. et } 3^{\mathrm{èm}} \mathrm{Ch} \text {, sous- } \\
\text { section II, Section } \\
\text { I, Première Partie } \\
\text { (pages 79-80) }\end{array}$ \\
\hline \multirow[t]{2}{*}{$3^{\prime} 51^{\prime \prime}$} & $26-32$ & $\begin{array}{l}\text { 1.C Dialogue entre Peña et Muñoz : le plan } \\
\text { de l'attaque au pont }\end{array}$ & \\
\hline & FE & & \\
\hline $5^{\prime} 52^{\prime \prime}$ & 33- 49 & $\begin{array}{l}\text { 1.D Réunion dans l'arrière boutique d'une } \\
\text { droguerie dans une ville : Carral prépare. Le } \\
\text { groupe sort dans la rue. }\end{array}$ & \\
\hline \multirow[t]{8}{*}{$10^{\prime} 18^{\prime \prime}$} & & 2. DEUXIÈME SEGMENT & \\
\hline & 50 & $\begin{array}{l}\text { TITRE : « Pendant ce temps, à Linas un } \\
\text { paysan qui a repéré le terrain d'aviation des } \\
\text { franquistes vient chercher un guide qui lui } \\
\text { fera traverser les lignes ennemies. » }\end{array}$ & \\
\hline & $51-54$ & $\begin{array}{l}\text { 2.A Linas. Plans sur la ville, jusqu'au } \\
\text { panneau du siège du Comité du Front } \\
\text { Populaire. }\end{array}$ & \\
\hline & FE & & \\
\hline & $55-68$ & $\begin{array}{l}\text { 2.B Réunion du Comité de Linas: le paysan et } \\
\text { sa mission (José Lado). }\end{array}$ & \\
\hline & FE & & \\
\hline & $69-105$ & $\begin{array}{l}\text { 2.C Carral et son groupe (avec Gonzales) } \\
\text { dans les rues : le combat dans la ville. Le } \\
\text { canon }\end{array}$ & $\begin{array}{l}2^{\text {ème }} \text { Ch., sous-section } \\
\text { I, Section I, Première } \\
\text { Partie, p. } 32\end{array}$ \\
\hline & FE & & \\
\hline
\end{tabular}

9 La division en segments est fondée sur le chapitre «Segmenter/Analyser» de L'analyse $d u$ film de Raymond Bellour (Bellour, 1979: 247-270)

$10 \mathrm{FE}=$ Fondu enchaînée; FauN= fermeture au noir ; OduN= Ouverture du noir ; V= volet de gauche à droite. 


\begin{tabular}{|c|c|c|c|}
\hline & $106-137$ & $\begin{array}{l}\text { 2.D. Rue de Móstoles : Carral et un ouvrier } \\
\text { lancent une voiture contre le canon, en } \\
\text { réussissant à le néutraliser. Ils meurent. }\end{array}$ & Ibid. \\
\hline & FE & & \\
\hline \multirow[t]{11}{*}{$17^{\prime} 40^{\prime \prime}$} & & 3. TROISIÈME SEGMENT & \\
\hline & 138 & $\begin{array}{l}\text { TITRE : « Les partisans républicains trouvent } \\
\text { une voiture chez un garagiste hors de la ville } \\
\text { et arrivent à Linas prêter main-forte aux } \\
\text { paysans qui doivent empêcher les franquistes } \\
\text { de passer par leur village et d'arriver au } \\
\text { pont. » }\end{array}$ & \\
\hline & 139 & $\begin{array}{l}\text { Ext. Linas. Gonzales et les partisans de la } \\
\text { ville arrivent à Linas. }\end{array}$ & \\
\hline & $\mathrm{V}$ & & \\
\hline & $140-145$ & $\begin{array}{l}\text { 3.A Intérieur Salle du Comité. Dialogue entre } \\
\text { Gonzales et le comité. }\end{array}$ & \\
\hline & FauN & & \\
\hline & $146-155$ & $\begin{array}{l}\text { 3.B Place devant le comité : la collecte des } \\
\text { récipients. }\end{array}$ & $\begin{array}{l}\text { 7ème Ch., Section I, } \\
\text { Deuxième Partie, p. } \\
311\end{array}$ \\
\hline & FE & & \\
\hline & 156 & $\begin{array}{l}\text { TITRE : «Et armés de ces récipients } \\
\text { hétéroclites remplis de dynamite, les paysans } \\
\text { combattirent les troupes franquistes et les } \\
\text { empêchèrent d'arriver jusqu'au pont qui } \\
\text { était leur seule ligne de communication avec } \\
\text { l'arrière...» }\end{array}$ & \\
\hline & FauN & & \\
\hline & OduN & & \\
\hline \multirow[t]{6}{*}{$21^{\prime} 55^{\prime \prime}$} & & 4. QUATRIÈME SEGMENT & \\
\hline & $157-164$ & $\begin{array}{l}\text { 4.A. Ext. Terrain d'aviation/ Essai de vol de } \\
\text { Schreiner et échec. Schreiner décide alors de } \\
\text { devenir mitrailleur. }\end{array}$ & $\begin{array}{l}\text { Troisième ch., sous- } \\
\text { section II, Section } \\
\text { I, Première Partie, } \\
\text { page72 }\end{array}$ \\
\hline & $165-174$ & $\begin{array}{l}\text { 4.B Int. Caserne. Attente pour le vol de } \\
\text { Schreiner. Les raisons de l'engagement de } \\
\text { chacun des aviateurs. }\end{array}$ & \\
\hline & $175-189$ & $\begin{array}{l}\text { 4.C Terrain aviation : Schreiner tombe. Il } \\
\text { demande à Pena d'être destiné à une autre } \\
\text { fonction. Ils se séparent }\end{array}$ & Ibid. \\
\hline & 190-202 & $\begin{array}{l}\text { 4.D Schreiner arrive sur le terrain d'exercice } \\
\text { des mitrailleur. Il essaie à tirer sur une cible } \\
\text { et réussit. }\end{array}$ & \\
\hline & FauN & & \\
\hline \multirow[t]{4}{*}{$26^{\prime} 35^{\prime \prime}$} & & 5. CINQUIÈME SEGMENT & \\
\hline & 203 & $\begin{array}{l}\text { TITRE: «Le paysan et son guide arrivent au } \\
\text { dernier village tenu par les franquistes. Ils } \\
\text { leur faut se renseigner sur l'itinéraire à suivre } \\
\text { pour traverser les lignes et parvenir au } \\
\text { terrain d'aviation des républicains.» }\end{array}$ & \\
\hline & FauN & & \\
\hline & OduN & & \\
\hline
\end{tabular}




\begin{tabular}{|c|c|c|c|}
\hline & 204-209 & $\begin{array}{l}\text { 5.A Extérieur café. Pio, le guide, convainc } \\
\text { José, le paysan, à entrer dans un café où ils } \\
\text { trouveront des amis, après un signa convenu }\end{array}$ & \\
\hline & $210-213$ & $\begin{array}{l}\text { 5.B Intérieur café. Le contact avec le patron } \\
\text { du café et le guide : le patron les ramène } \\
\text { dans le jardin. }\end{array}$ & \\
\hline & $214-220$ & $\begin{array}{l}\text { 5.C Le patron se révèle un ennemi qui tue le } \\
\text { guide. José arrive à tuer le traître. }\end{array}$ & \\
\hline & FauN & & \\
\hline & OduN & & \\
\hline \multirow[t]{3}{*}{$30^{\prime} 04^{\prime \prime}$} & & 6. SIXIÈME SEGMENT & \\
\hline & $221-232$ & $\begin{array}{l}\text { 6.A Terrain d'aviation. Dialogue entre le } \\
\text { paysan et Peña : José indiquera le village à } \\
\text { bombarder lors de la prochaine incursion. } \\
\text { dialogue entre Peña et Attignies : ce dernier } \\
\text { est nommé commissaire politique de } \\
\text { l'escadrille }\end{array}$ & $\begin{array}{l}\text { Ch. III, Troisième } \\
\text { Partie, pages } 442-443\end{array}$ \\
\hline & $\mathrm{V}$ & & \\
\hline \multirow[t]{6}{*}{$33^{\prime} 16^{\prime \prime}$} & $233-235$ & $\begin{array}{l}\text { 6.B Peña organise l'incursion avec le chef } \\
\text { d'un deuxième avion qui devra bombarder } \\
\text { un pont. }\end{array}$ & \\
\hline & FauN & & \\
\hline & OduN & & \\
\hline & $236-242$ & $\begin{array}{l}\text { 6.C Attignies et Peña visitent l'atelier de } \\
\text { réparation des avion, puis il prennent } \\
\text { une voiture et se dirigent vers les comités } \\
\text { républicains des paysans }\end{array}$ & \\
\hline & FauN & & \\
\hline & OduN & & \\
\hline \multirow[t]{7}{*}{$36^{\prime} 27^{\prime \prime}$} & & 7. SEPTIÈME SEGMENT & \\
\hline & 243 & $\begin{array}{l}\text { TITRE: «Pour permettre l'envol de nuit, } \\
\text { le Commandant et le Commissaire vont } \\
\text { demander aux paysans des villages voisins } \\
\text { de leur prêter des autos dont les phares } \\
\text { marqueront les limites du terrain.» }\end{array}$ & \\
\hline & $\begin{array}{l}\text { Surimpression } \\
\text { du Titre sur } \\
\text { plans 244-245 }\end{array}$ & $\begin{array}{l}\text { La voiture de Peña marche jusqu'à arriver } \\
\text { devant un immeuble d'une ferme. }\end{array}$ & \\
\hline & $246-255$ & $\begin{array}{l}\text { 7.A Int. Comité des paysans, Jour. Le } \\
\text { Commandant Peña demande des autos, il en } \\
\text { obtient trois. Il se remet en route. Nuit }\end{array}$ & $\begin{array}{l}\text { Ch. III, Troisième } \\
\text { Partie, page } 445-446\end{array}$ \\
\hline & FE & & \\
\hline & 256 & $\begin{array}{l}\text { La voiture de Peña traverse un poste de } \\
\text { contrôle }\end{array}$ & \\
\hline & FE & & \\
\hline \multirow[t]{5}{*}{$38^{\prime} 53^{\prime \prime}$} & $257-266$ & $\begin{array}{l}\text { 7.B Int./Ext. Comité d'un autre village, nuit. } \\
\text { Peña demande d'autres voitures. Il se remet } \\
\text { en marche avec Attignies. }\end{array}$ & Ibid. \\
\hline & FE & & \\
\hline & 267 & La voiture voyage dans la nuit & \\
\hline & FauN & & \\
\hline & OduN & & \\
\hline
\end{tabular}




\begin{tabular}{|c|c|c|c|}
\hline $40^{\prime} 16^{\prime \prime}$ & $268-273$ & $\begin{array}{l}\text { 7.C Ext. Premier comité (celui de 7.A), nuit. } \\
\text { Peña et Attignies regardent les paysans } \\
\text { qui transportent les victuailles pour le } \\
\text { ravitaillement sur leur dos, pour laisser } \\
\text { les voitures disponibles pour le terrain } \\
\text { d'aviation }\end{array}$ & Ibid., page 447 \\
\hline & FauN & & \\
\hline & OduN & & \\
\hline \multirow[t]{7}{*}{$40^{\prime} 58^{\prime \prime}$} & 274 & $\begin{array}{l}\text { La voiture du Commandant et du } \\
\text { Commissaire voyage encore dans la nuit }\end{array}$ & \\
\hline & FE & & \\
\hline & 275 & Encore la voiture sur la route & \\
\hline & FE & & \\
\hline & $276-283$ & $\begin{array}{l}\text { 7.D Ext. terrain d'aviation, nuit. Peña et } \\
\text { Attignies arrivent, les autos devant marquer } \\
\text { la limite de la piste sont en place. }\end{array}$ & Ibid., page 448 \\
\hline & FauN & & \\
\hline & OduN & & \\
\hline \multirow[t]{6}{*}{$42^{\prime} 16^{\prime \prime}$} & & 8. HUITIÈME SEGMENT & \\
\hline & 284-301 & $\begin{array}{l}\text { 8.A Ext. Terrain d'aviation, nuit. Les phares } \\
\text { des autos s'allument.Le premier avion } \\
\text { décolle. }\end{array}$ & Ibid., page 449 \\
\hline & FE & & \\
\hline & $302-305$ & Décollage du deuxième avion. & Ibid. \\
\hline & FauN & & \\
\hline & OduN & & \\
\hline \multirow[t]{3}{*}{$44^{\prime} 25^{\prime \prime}$} & & 9. NEUVIÈME SEGMENT & \\
\hline & $306-422$ & $\begin{array}{l}\text { 9.A Int. Premier avion, jour (aube). Peña } \\
\text { et le paysan, qui reconnaît le terrain des } \\
\text { franquiste. Le bombardement et la bataille } \\
\text { aerienn contre la chasse franquiste. }\end{array}$ & Ibid., pp. 450-455 \\
\hline & $423-533$ & $\begin{array}{l}\text { 9.B Int. Deuxième avion, jour. Le deuxième } \\
\text { avion va accomplir la mission de } \\
\text { bombardement d'un pont et va se battre } \\
\text { contre les avions ennemis. L'avion s'écrase } \\
\text { contre la montagne. }\end{array}$ & Ibid., page $456-458$ \\
\hline \multirow[t]{4}{*}{$56^{\prime} 03^{\prime \prime}$} & & 10. DIXIÈME SEGMENT & \\
\hline & $\begin{array}{l}534 \\
\text { surimpression } \\
\text { du Titre sur le } \\
\text { plan } 535\end{array}$ & $\begin{array}{l}\text { TITRE: «Le premier avion est rentré à } \\
\text { sa base. Le Commandant, avisé par le } \\
\text { téléphone qu'on a vu un appareil tomber } \\
\text { dans la montagne, part à la recherche de ses } \\
\text { hommes» }\end{array}$ & \\
\hline & $535-560$ & $\begin{array}{l}\text { 10.A Ext./int. maison du postier du village } \\
\text { de Linares, proche de la montagne. Peña } \\
\text { découvre que ses hommes ont reçu les } \\
\text { premiers secours et qui descendent de la } \\
\text { montagne vers le village de Valdelinares, il } \\
\text { apprête une ambulance et part. }\end{array}$ & Ibid., pages $459-462$ \\
\hline & FE & & \\
\hline $58^{\prime} 25^{\prime \prime}$ & $561-564$ & $\begin{array}{l}\text { 10.B Ext. Montagne : descente du corps de } \\
\text { Marquez, blessé au visage. Les villageois } \\
\text { parcourent les chemins vers la montagne, à } \\
\text { travers les oliviers. }\end{array}$ & Ibid., pp. 467 suiv. \\
\hline
\end{tabular}




\begin{tabular}{|l|l|l|l|}
\hline & FE & & \\
\hline $59^{\prime} 42^{\prime \prime}$ & 565 & Encore un chemin de la montagne. & \\
\hline & FE & & \\
\hline & $566-567$ & $\begin{array}{l}\text { Les paysans et les aviateurs, un des blessés } \\
\text { monte sur un âne. }\end{array}$ & \\
\hline & FE & $\begin{array}{l}\text { 10. C Ext. Village. La réunion des anciens du } \\
\text { village. }\end{array}$ & \\
\hline & 568 & $\begin{array}{l}\text { Int. maison village. Les anciens décident } \\
\text { d'aller accueillir les blessés. }\end{array}$ & \\
\hline & FE & $\begin{array}{l}\text { 10.D Les blessés sont transportés sur des } \\
\text { civières par les paysans. Peña parle avec } \\
\text { Marquez et avec Schreiner. Le cortège arrive } \\
\text { au village, les paysans saluent les blessés }\end{array}$ & Ibid., 467-474 \\
\hline 1 1h01'08' & $572-626$ & & \\
\hline $1 h 08^{\prime} 04^{\prime \prime}$ & FauN & & \\
\hline $1 \mathrm{~h} 08^{\prime \prime 17} \prime$ & & & \\
\hline
\end{tabular}

